\title{
An innovative robotic platform for magnetically-driven painless colonoscopy
}

\author{
Federico Bianchi ${ }^{1 *}$, Gastone Ciuti ${ }^{*}$, Anastasios Koulaouzidis ${ }^{2}$, Alberto Arezzo ${ }^{3}$, Danail Stoyanov ${ }^{4}$, \\ Sebastian Schostek ${ }^{5}$, Calogero Maria Oddo ${ }^{1}$, Arianna Menciassi ${ }^{1}$, Paolo Dario ${ }^{1}$ \\ ${ }^{1}$ The BioRobotics Institute, Scuola Superiore Sant'Anna, Pisa, Italy; ${ }^{2}$ Endoscopy Unit, The Royal Infirmary of Edinburgh, Edinburgh, Scotland, \\ UK; ${ }^{3}$ Department of Surgical Sciences, University of Torino, Torino, Italy; ${ }^{4}$ Centre for Medical Image Computing and the Department of Computer \\ Science, University College London, London, UK; ${ }^{5}$ Ovesco Endoscopy AG, Tübingen, Germany \\ Contributions: (I) Conception and design: All authors; (II) Administrative support: None; (III) Provision of study materials or patients: None; (IV) \\ Collection and assembly of data: None; (V) Data analysis and interpretation: None; (VI) Manuscript writing: All authors; (VII) Final approval of \\ manuscript: All authors. \\ *These authors contributed equally to this work. \\ Correspondence to: Gastone Ciuti. The BioRobotics Institute, Scuola Superiore Sant'Anna, viale Rinaldo Piaggio 34, Pontedera 56025, Pisa, Italy. \\ Email: gastone.ciuti@santannapisa.it.
}

\begin{abstract}
Colorectal cancer (CRC) represents a significant medical threat with a dramatic impact on the healthcare system with around 1.3 million patients worldwide, causing more than 700 thousand deaths annually. A key-aspect to successful and cost-effective disease management is represented by the early detection of CRC at asymptomatic stage. For this reason, population screening is highly recommended for patients older than 50 years or at high risk for familiarity. Currently, the standard endoscopic techniques do not meet this need. In recent years, innovative endoscopic robotic techniques and active locomotion devices have been developed as alternatives to conventional colonoscopy. The magnetically-driven robotic platform, presented by the authors, is conceived to perform less invasive and more comfortable colonoscopy with the aim to promote screening campaigns for detection of early colorectal neoplasm.
\end{abstract}

Keywords: Gastrointestinal endoscopy (GI endoscopy); painless endoscopy; robotic-aided colonoscopy; magnetically-driven robotic platform; soft-tether colonoscopic capsule

Submitted Aug 16, 2017. Accepted for publication Aug 29, 2017.

doi: 10.21037/atm.2017.09.15

View this article at: http://dx.doi.org/10.21037/atm.2017.09.15

\section{Medical rationale and clinical needs: the colonoscopy case}

Cancer is a leading cause of death, counting about 8.8 million deaths worldwide and representing $15.6 \%$ of global deaths in 2015 (1). In 2012, 14.1 million new cancer cases, 8.2 million cancer deaths and 32.6 million people living with cancer (within 5 years of diagnosis) were recorded worldwide (2). More alarmingly, death from cancer is projected to rise over 13 million by 2030 (3). Colorectal cancer (CRC) ranks as the third most common among other cancers and remains a significant medical threat with a dramatic impact on healthcare systems (4-6); CRC affects around 1.3 million individuals worldwide, causing more than 700 thousand deaths annually (2). CRC represents the third and fourth most prevalent cancer in term of number of patients and number of deaths worldwide, respectively. Moreover, it is the second most common cancer affecting European women and the third most common for European men (2). Early CRC detection is a key issue to successful and cost-effective disease management as the 5 -year survival rate decreases with the progress of the CRC stage, i.e., $94 \%$ when the diagnosis is made at an early stage, while plummeting to $11 \%$ with advanced disease (cancer stage IV) (7). For this reason, regular screening is highly recommended for patients older than 50 years of age or for 
those in higher risk groups such as individuals with a family history of CRC $(6,8,9)$.

However, the efficacy of the CRC screening programmes is limited due to low participation rate, since the relevant diagnostic procedures are associated with several drawbacks: (I) invasiveness, i.e., sigmoidoscopy or colonoscopy; (II) unpleasant preparation phase, i.e., bowel purge and/or fear of embarrassment during the procedure; and (III) fear of discomfort/pain and/or need for sedation. Currently, there are three main techniques for CRC diagnosis: (I) conventional colonoscopy; (II) computed tomography (CT)-scan or virtual colonoscopy; and (III) wireless capsule endoscopy (WCE). Conventional colonoscopy remains the reference standard technique for diagnosis and treatment of preneoplastic and neoplastic pathology due to its capability to directly visualize the inner surface of the colon, acquire biopsies and treat pathologies in the same session (10). However, colonoscopy requires bowel preparation and, despite the advent of more comfortable colonoscope models, it can still cause significant pain/discomfort that requires intravenous sedation and/or analgesia or even general anaesthesia. On the other hand, CT-scan colonoscopy represents the first alternative to traditional endoscopic techniques. The images produced during the CT-scan examination can be reformed to generate a $3 \mathrm{D}$ reconstruction of the colon lumen and a virtual colonoscopy can be performed without causing any patient discomfort hence without the need for sedation $(11,12)$. Nevertheless, CT-scan colonoscopy does not allow the direct treatment of any lesion, it requires bowel preparation and it is limited in inspection sensitivity and efficiency for small lesions or flat polyps. Lastly, WCE represents the most relevant progress in endoscopic technology, but with an insufficient view of the inner colonic walls due to the inability to control the capsule itself (13). It is designed to allow direct inspection of the completely gastrointestinal (GI) tract in a minimally invasive manner without the need of sedation, patient discomfort or pain. However, the main limitations of current WCE technology are: (I) the low inspection sensitivity and efficiency, arising due to its passive locomotion; (II) lack of bowel distension; and (III) low imaging quality $(14,15)$. In recent years, innovative endoscopic robotic techniques and active locomotion devices have been developed in order to reduce pain and patient discomfort without lacking in diagnostic accuracy and procedure reliability. In this framework, a magnetically-driven robotic platformdesigned and developed within the Endoscopic versatile robotic guidance, diagnosis and therapy of magnetic-driven soft-tethered endoluminal robots European Project (H2020ICT-24-2015-GA: 688592)—may represent an alternative technique to conventional colonoscopy, being designed to perform less invasive and less uncomfortable colonoscopy with the aim of promoting mass screening campaigns for early CRC detection (16).

\section{Conventional methods and new devices: state of the art}

Semi-flexible endoscopes for inspection of the GI tract were conceived in 1868 by Wolf and Schindler, who became the fathers of modern GI endoscopy. Nowadays, flexible scopes are considered the standard endoscopic tool enabling effective and reliable operation through different segments of the GI tract with screening, diagnostic and also therapeutic/surgical capabilities. The effectiveness of the use of conventional colonoscopes is mainly due to their capability of performing diagnosis and treatment in a controlled manner. The endoscopist manually inserts and pushes the colonoscope along the colon, steering its tip by a combination of shaft torque and wheels in the operator control handle. The scope is flexible enough so that its tip gets up to cecum but stretching the physiological curves of the colon due to its stiffness. The modification of the natural colon shape and the stretching of the mesentery, together with lumen distension due to gas insufflation, are the main causes of discomfort and potential tissue damage or even perforation $(17,18)$, with direct consequence on patient discomfort, need for sedation and fear of attending the screening programme. However, the stiffness of the colonoscope shaft is an intrinsically necessary feature permitting manual control and endoscopic navigation, representing a "rear-wheel drive" navigation approach. The insertion shaft must be stiffer if compared to the colon tissue to prevent the curling/wrinkling of the scope tube along the colon. Furthermore, current colonoscopes have been poorly optimized towards ergonomy and control effectiveness for the operators, who can be subject to musculoskeletal injuries such as carpal tunnel and de Quervain's syndrome (19). This is because the force/pressure to insert, push, move and orient the colonoscope tip is an exclusively manual task. At present, the endoscopist's mental and physical resources are essentially drained by the heavy manual workload (20), resulting in reduced attention spans and frequently diagnostic efficiency. 
On the other hand, virtual colonoscopy represents the best alternative to conventional colonoscopy to perform diagnosis for polyps and cancer screening in the colon. It is a non-invasive external technique, it requires no sedation, and it can be completed in a much shorter time (21). A very small flexible tube is passed into rectum to allow air to be pumped into the colon for distending the inner surfaces. Thereafter, the CT-scanner acquires the cross-section images produced by $\mathrm{X}$-rays in both prone and supine positions with a thin collimator. CT colonoscopy is able to identify lesions behind haustral folds and beyond colonic bends because of its ability to provide an endoluminal view of the colon in both forward and reverse directions and its ability to represent the colon in both two-dimensional and three-dimensional perspectives (21). In addition, it has the ability to visualize the colon lumen even in the presence of obstructions that would not undergo a complete inspection with the use of a conventional colonoscope. The key-factor to guarantee the success of the CT examination is a good bowel preparation with laxatives, because residual faecal material can lead to false positives. Virtual colonoscopy has certain limitations: (I) the inability to acquire biopsy and to treat lesions; (II) the exposition of the patient to ionizing $\mathrm{X}$-ray radiation; (III) the inaccurate visualization of the colon walls due to insufficient insufflation of the lumen; and (IV) the inability to detect small lesions due to the spatial accuracy of the CT-scanner, such as flat polyps. Indeed, Johnson et al. (22) in 2003 reports that polyp sensitivity in virtual colonoscopy for polyps $6-9$ and $\geq 10 \mathrm{~mm}$ in diameter ranged from $20 \%$ to $44 \%$ and $60 \%$ to $78 \%$, respectively.

The third main endoscopic diagnostic technology is the WCE, which represents the most relevant technological progress of conventional GI endoscopy. WCE system is composed of three main components: (I) the endoscopic capsule built with a disposable plastic shell with several modules embedded: vision sensor, illumination, batteries, integrated electronic transmitter, and antenna; (II) an external sensing system to collect the data coming from the embedded telemetry module and for capsule location detection; and (III) a real-time image stream viewer and dedicated software for diagnosis $(16,23)$. The first capsule model, produced by Given Imaging Ltd. (Yokneam Illit, Israel) for the small intestine, was approved by Food and Drug Administration in 2001. After the introduction of this disruptive technology, several companies produced other WCE models such as Olympus, Co. (Tokyo, Japan) with the EndoCapsule, and IntroMedic, Co., Ltd. (Seoul, South Korea) with the MiroCam. Since 2006, when the PillCam
Colon by Given Imaging Ltd. has received the CE mark, WCE has been used for colonic inspection. However, the main application field of WCE remains the examination of the small bowel as it does not require either air insufflation or water irrigation to distend the lumen for proper visualization of the mucosa and because small bowel is not approachable with conventional endoscopes. Instead, the large bowel requires an unnatural distention of the inner wall to allow a proper visualization and locomotion of the WCE due to the fact that the tract is naturally collapsed. Furthermore, the inability of capsule orientation and navigation control does not allow for a correct inspection of the entire inner wall of the colon (24). However, this innovative technology has been met with a great deal of enthusiasm by patients due to the significant reduction of pain, invasiveness and examination discomfort.

In recent years, colonoscopic technology has been refined and, nowadays, many research teams are working on technology enhancements to combine the low invasiveness and high patient tolerability of the WCE with the ability to perform diagnosis and treatment in the same section (25). Significant examples of innovative smart endoscopes are: (I) Endotics (EraEndoscopy s.r.l., Peccioli, Italy) (26); (II) CathCam (Ethicon, Cincinnati, OH, USA) (27); (III) Aer-O-Scope (GI View Ltd., Ramat Gan, Israel) (28); (IV) NeoGuide (Neoguide Systems Inc., Los Gatos, CA, USA) (29); (V) Invendo SC20 (Invendo Medical GmbH, Kissing, Germany) (30,31); (VI) Colonosight (Stryker Corp., Kalamazoo, MI, USA) (32); and (VII) EndoEase (Spirus Medical, LLC, Bridgewater, MA, USA) (33).

\section{The magnetically-driven robotic platform: a new solution exists}

The Endoscopic versatile robotic guidance, diagnosis and therapy of magnetic-driven soft-tethered endoluminal robots Project aims to develop an active colonoscopic platform for teleoperated robotic guidance of a painless, innovative, smart, and soft-tethered device, in order to achieve, by effective mass screening, accurate and reliable diagnosis and eventual therapy of colonic pathologies. Navigation, diagnosis, therapy modules and safety strategies for human-robot interaction/cooperation will represent the main components of the proposed robotic platform, which will allow for a magnetically-actuated and painless colonoscopic procedure. The platform will offer benefit both in terms of reduction of patient's pain and discomfort and in terms of reduction of endoscopist's physical strain, 


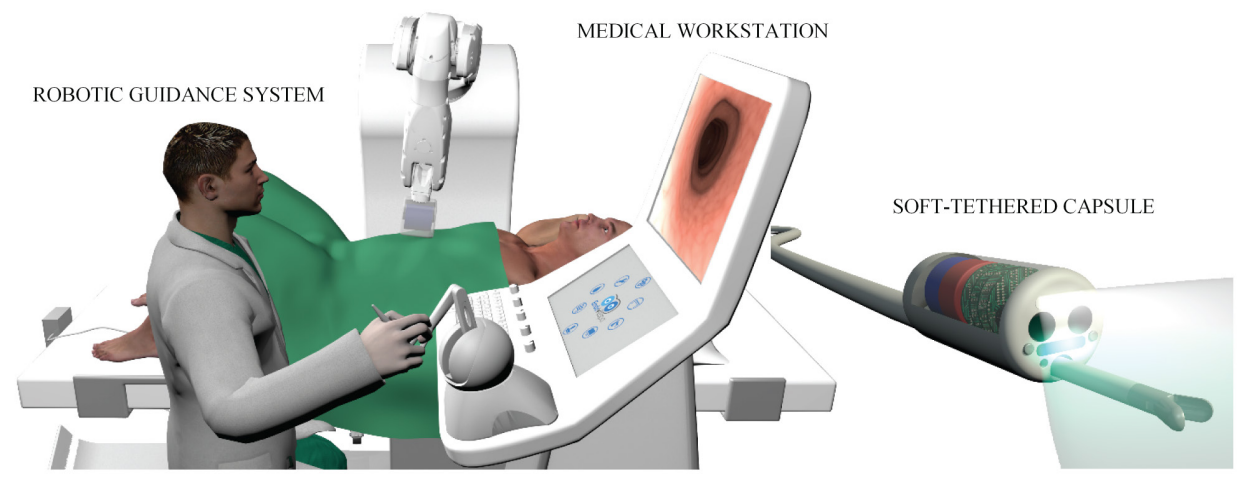

Figure 1 Rendering of the magnetically-driven robotic platform.

ergonomics and mechanical load.

Three main modules, as shown in Figure 1, compose the platform: (I) a soft-tethered stereoscopic capsule; (II) a robotic guidance system; and (III) the medical workstation. The smart soft-tethered stereoscopic capsule, able to perform painless diagnosis and treatment, embeds the same functionalities of a standard colonoscope and it is improved by adopting a stereoscopic vision module. The advanced design of the soft-tether guarantees the capability of the robotic capsule to proceed along the colonic tract without stretching the mesentery and, consequently, aiming at reducing pain, patient discomfort, looping, and perforation risk. The robotic guidance system includes a robotic arm, a dedicated setting module designed to increase the ergonomic conditions of the endoscopist, and an integrated localization module compatible with highintensity magnetic field sources for locomotion. The internal permanent magnet, embedded onto the capsule, is driven by an external permanent magnet connected to the robotic arm, which is able to move the magnet above the entire abdomen of the patient. Magnetic navigation turns out to be one of principal alternative locomotion approach to manual locomotion; it represents the change from the "rear-wheel drive" to the "front-wheel drive" method. The endoscopist guides directly the capsule with high controllability and reliability thanks to an accurate localization method. The intuitive medical workstation controls the entire platform in an easy way. Teleoperated robotic endoscopy with an ergonomically-optimized workstation design may represent a viable alternative to reduce or even eradicate endoscopy-related musculoskeletal injuries, as already presented and discussed in several studies (19). The design of the magnetically-driven robotic platform will aim at increasing the ergonomic conditions of both endoscopist and patient. In particular, the teleoperated control modality and the dedicated setting module may reduce strain and mechanical load on the endoscopist including an ergonomic position at the workstation. Furthermore, the software development of a computer integrated support, such as convolution neural networks for automatic polyp detection support (34) and algorithms for mapping the colon and the position of the cameras within it, may reduce mental and physical demands thus allowing the endoscopist to perform a more efficient and accurate procedure.

\section{Conclusions and future perspectives}

Teleoperated robotic colonoscopy is more and more becoming a forward-looking solution for colon inspection and basic interventional activity thanks to an appropriate design optimized to reduce physical and mental load for the endoscopist and to achieve less invasive and less uncomfortable diagnosis and treatment. In particular, magnetically-driven colonoscopy, achieved with the robotic platform presented by the authors, turns the entire locomotion approach from a "rear-wheel drive" to a "frontwheel drive" paradigm. The magnetic approach guarantees high control of the capsule and, combined with humanrobot interaction strategies, drastically reduces invasiveness and significantly improves diagnostic accuracy and reliability.

In the future, the magnetically-driven robotic platform, presented by the authors, is expected to play a major role in the robotic colonoscopy scene for the ability to integrate the main and most effective features of both conventional colonoscopy and WCE in an intuitive, comfortable, magnetically-driven, soft-tethered robotic capsule solution. 


\section{Acknowledgements}

The authors thank all the collaborators of the EU project. Funding: The work described in this paper was supported by the European Commission within the framework of the endoscopic versatile robotic guidance, diagnosis and therapy of magnetic-driven soft-tethered endoluminal robots Project-H2020-ICT-24-2015 (EU Project-G.A. number: 688592).

\section{Footnote}

Conflicts of Interest: The authors have no conflicts of interest to declare.

\section{References}

1. International-Agency-for-Research-on-Cancer. Global Health Observatory (GHO) data, NCD mortality and morbidity. Available online: http://www.who.int/gho/ncd/ mortality_morbidity/en/

2. International-Agency-for-Research-on-Cancer. Estimated Incidence, Mortality and Prevalence Worldwide in 2012. Available online: http://globocan.iarc.fr/Pages/fact_sheets_ cancer.aspx

3. Wang X, Di Natali C, Beccani M, et al. Novel medical wired palpation device: A validation study of material properties. Solid-State Sensors, Actuators and Microsystems (TRANSDUCERS \& EUROSENSORS XXVII), 2013 Transducers \& Eurosensors XXVII: The 17th International Conference. 2013, IEEE.

4. Ghanouni A, Smith SG, Halligan S, et al. Public preferences for colorectal cancer screening tests: a review of conjoint analysis studies. Expert Rev Med Devices 2013;10:489-99.

5. Schreuders EH, Ruco A, Rabeneck L, et al. Colorectal cancer screening: a global overview of existing programmes. Gut 2015;64:1637-49.

6. Senore C, Inadomi J, Segnan N, et al. Optimising colorectal cancer screening acceptance: a review. Gut 2015;64:1158-77.

7. Lansdorp-Vogelaar I, van Ballegooijen M, Zauber AG, et al. Effect of rising chemotherapy costs on the cost savings of colorectal cancer screening. J Natl Cancer Inst 2009;101:1412-22.

8. Armaroli P, Villain P, Suonio E, et al. European Code against Cancer: cancer screening. Cancer epidemiology 2015;39:S139-52.
9. Hassan C, Rossi PG, Camilloni L, et al. Meta-analysis: adherence to colorectal cancer screening and the detection rate for advanced neoplasia, according to the type of screening test. Aliment Pharmacol Ther 2012;36:929-40.

10. Leung WC, Foo DC, Chan TT, et al. Alternatives to colonoscopy for population-wide colorectal cancer screening. Hong Kong Med J 2016;22:70-7.

11. Burling D; International Collaboration for CT colonography Standards. CT colonography standards. Clin Radiol 2010;65:474-80.

12. Lichan H, Kaufman A, Yi-Chih W, et al. 3D virtual colonoscopy. Proceedings 1995 Biomedical Visualization. Atlanta, GA, USA, 199530 Oct-3 Nov 1995.

13. Van Gossum A, Munoz-Navas M, Fernandez-Urien I, et al. Capsule Endoscopy versus Colonoscopy for the Detection of Polyps and Cancer. N Engl J Med 2009;361:264-70.

14. Iddan G, Meron G, Glukhovsky A, et al. Wireless capsule endoscopy. Nature 2000;405:417.

15. Siddiqui AA, Fayiga Y, Huerta S. The role of endoscopic ultrasound in the evaluation of rectal cancer. Int Semin Surg Oncol 2006;3:36.

16. Ciuti G, Caliò R, Camboni D, et al. Frontiers of robotic endoscopic capsules: a review. Journal of Micro-Bio Robotics 2016;11:1-18.

17. Lohsiriwat V. Colonoscopic perforation: Incidence, risk factors, management and outcome. World J Gastroenterol 2010;16:425-30.

18. Kavic SM, Basson MD. Management of complications of colonoscopy. In: Holzheimer RG, Mannick JA. editors. Surgical Treatment: Evidence-Based and ProblemOriented. Munich: Zuckschwerdt, 2001.

19. Yung DE, Banfi T, Ciuti G, et al. Musculoskeletal injuries in gastrointestinal endoscopists: a systematic review. Expert Rev Gastroenterol Hepatol 2017;11:939-47.

20. Rahman I, Boger P, Patel P. OC-012 The Effect of Mental Workload Experienced During Colonoscopy on Endoscopists Performance. Gut 2016;65:A9-A.

21. Heiken JP, Peterson CM, Menias CO. Virtual colonoscopy for colorectal cancer screening: current status: Wednesday 5 October 2005, 14:00-16:00. Cancer Imaging 2005;5:S133-S9.

22. Johnson CD, Harmsen WS, Wilson LA, et al. Prospective blinded evaluation of computed tomographic colonography for screen detection of colorectal polyps. Gastroenterology 2013;125:311-9.

23. Wang A, Banerjee S, Barth BA, et al. Wireless capsule endoscopy. Gastrointestinal Endoscopy 2013;78:805-15.

24. Koulaouzidis A, Iakovidis DK, Karargyris A, et al. Wireless 
endoscopy in 2020: Will it still be a capsule? World J

Gastroenterol 2015;21:5119-30.

25. Sliker LJ, Ciuti G. Flexible and capsule endoscopy for screening, diagnosis and treatment. Expert Rev Med Devices 2014;11:649-66.

26. Tumino E, Sacco R, Bertini M, et al. Endotics system vs colonoscopy for the detection of polyps. World $\mathrm{J}$ Gastroenterol 2010;16:5452.

27. Fritscher-Ravens A, Fox S, Swain C, et al. CathCam guide wire-directed colonoscopy: first pilot study in patients with a previous incomplete colonoscopy. Endoscopy 2006;38:209-13.

28. Arber N, Grinshpon R, Pfeffer J, et al. Proof-of-concept study of the Aer-O-scope ${ }^{\mathrm{TM}}$ omnidirectional colonoscopic viewing system in ex vivo and in vivo porcine models. Endoscopy 2007;39:412-7.

29. Eickhoff A, Van Dam J, Jakobs R, et al. Computer-assisted colonoscopy (the NeoGuide Endoscopy System): results of the first human clinical trial ("PACE study"). Am J

Cite this article as: Bianchi F, Ciuti G, Koulaouzidis A, Arezzo A, Stoyanov D, Schostek S, Oddo CM, Menciassi A, Dario P. An innovative robotic platform for magnetically-driven painless colonoscopy. Ann Transl Med 2017;5(21):421. doi: 10.21037/ atm.2017.09.15
Gastroenterol 2007;102:261-6.

30. Groth S, Rex DK, Rösch T, et al. High cecal intubation rates with a new computer-assisted colonoscope: a feasibility study. Am J Gastroenterol 2011;106:1075-80.

31. Rösch T, Adler A, Pohl H, et al. A motor-driven singleuse colonoscope controlled with a hand-held device: a feasibility study in volunteers. Gastrointest Endosc 2008;67:1139-46.

32. Shike M, Fireman Z, Eliakim R, et al. Sightline ColonoSight system for a disposable, power-assisted, nonfiber-optic colonoscopy (with video). Gastrointest Endosc 2008;68:701-10.

33. Schembre DB, Ross AS, Gluck MN, et al. Spiral overtubeassisted colonoscopy after incomplete colonoscopy in the redundant colon. Gastrointest Endosc 2011;73:515-9.

34. Esteva A, Kuprel B, Novoa RA, et al. Dermatologist-level classification of skin cancer with deep neural networks. Nature 2017;542:115-8. 